Relations industrielles

Industrial Relations

\title{
Le Rapport Gill
}

\section{Jacques St-Laurent}

Volume 18, numéro 1, janvier 1963

URI : https://id.erudit.org/iderudit/1021457ar

DOI : https://doi.org/10.7202/1021457ar

Aller au sommaire du numéro

Éditeur(s)

Département des relations industrielles de l’Université Laval

ISSN

0034-379X (imprimé)

1703-8138 (numérique)

Découvrir la revue

Citer ce document

St-Laurent, J. (1963). Le Rapport Gill. Relations industrielles / Industrial

Relations, 18(1), 85-88. https://doi.org/10.7202/1021457ar

Tous droits réservés (C Département des relations industrielles de l’Université Laval, 1963
Ce document est protégé par la loi sur le droit d'auteur. L'utilisation des services d'Érudit (y compris la reproduction) est assujettie à sa politique d'utilisation que vous pouvez consulter en ligne.

https://apropos.erudit.org/fr/usagers/politique-dutilisation/ 
Sur le plan du commerce extérieur, on recommande des mesures pour accroître le commerce extérieur et une assistance canadienne aux pays sous-développés de $2 \%$ du revenu national. Ces objectifs sont désirables en autant qu'il s'agit d'accroissement des exportations et non des importations et que l'aide consiste dans des biens fabriqués au Canada qui ne soient pas des moyens de subventionner indirectement des industries marginales. Ces mesures mettent en cause la politique commerciale et tarifaire qui est désirable pour le Canada mais ne contiennent aucune proposition précise quant à ce que devrait être cette politique.

Le postulat de base de notre évaluation du contenu de la pensée économique syndicale consiste dans la proposition que le syndicalisme doit jouer un rôle positif dans l'élaboration de la politique économique, particulièrement dans le contexte du Canada français, puisque les effets d'une planification toucheront le niveau de vie de la classe ouvrière. Sur ce point, la contribution du Congrès de la C.S.N. fut plutôt faible. Ce n'est pas là un blâme pour cette centrale syndicale. Au moins y a-t-on posé des problèmes importants en termes analytiques valables tandis que le rapport du Conseil d'Orientation Economique basé sur des moyens plus considérables et un accès plus grand à l'expertise économique n'a inclus que des généralités et même des planitudes quand ce ne sont pas des propositions qui ne sont pas prouvées telle que la suivante: "lintégration poussée de l'économie canadienne à l'économie américaine est responsable dans une large mesure de la paresse de l'économie canadienne $\gg .^{3}$ Sur ce plan, les travaux de contenu économique du Congrès de la C.S.N. souffrent bien la comparaison.

\section{LE RAPPORT GILL}

\section{Jacques St-Laurent}

1 - L'intérêt du Rapport Gill ${ }^{1}$ est triple: il permet d'apprécier rapidement l'évolution du régime d'assurance-chômage au cours de ses vingt et une années d'existence; il présente certaines statistiques qui soulignent de façon dramatique l'accroissement du chômage depuis 1954-55; il offre enfin des suggestions intéressantes concernant le règlement des problèmes financiers des chômeurs.

2 - Fondamentalement, les suggestions des commissaires consistent en un ré-arrangement du fardeau financier occasionné par la nécessité d'assurer un revenu à certains de ceux qui n'ont pas de travail. De

(3) Le Conseil d'Orientation Economique du Québec, Documents de base en vue de la planification $\gg$, reçus et adoptés en essemblée régulière du Conseil d'Orientation Economique du Québec, le 21 septembre 1962.

(1) Rıpport du Comité d'Enquête relatif da la Loi sur lassurance-chômage, Ottawa, novembre 1962. 
plus, leurs suggestions, si elles étaient toutes acceptées, permettraient à un plus grand nombre de travailleurs de recevoir un revenu plus longtemps alors qu'ils sont en chômage.

3 - La ré-allocation du fardeau financier suggérée par les commissaires nous semble plus équitable. Elle consiste à restreindre les responsabilités de la Caisse d'assurance-chômage et à augmenter d'autant celles du gouvernement. C'est ainsi qu'une fraction plus considérable du fardeau financier occasionné par l'octroi de revenus aux chômeurs serait supportée par le gouvernement à même ses revenus généraux. Ne serait réservé à la Caisse que l'octroi des revenus aux chômeurs qui le sont pendant une courte période et de façon irrégulière.

4 - C'est là, dans un certain sens, renverser la vapeur et redonner à la Caisse sa véritable nature de caisse d'assurance. En effet, dans le passé, le gouvernement, par de nouveaux règlements, a imposé à la Caisse des largesses de plus en plus considérables, soit parce que l'actif de la Caisse augmentait rapidement, soit parce que les problèmes sociaux posés par le chômage devenaient de plus en plus pressants. En fait, les commissaires remarquent que toutes les modifications apportées à la Loi d'assurance-chômage «tendaient à élargir le champ d'application, à prolonger les prestations et à réduire les conditions d'admissibilité \$. ${ }^{2}$

Leurs suggestions consistent à restreindre le champ d'application, raccourcir les prestations et augmenter les conditions d'admissibilité.

5 - C'est ainsi, entre autres choses, que la durée maximum des prestations en provenance de la Caisse (car les commissaires suggèrent d'autres prestations provenant directement des revenus du gouvernement) serait réduite de 52 à 26 semaines, que seraient exclus les travailleurs qui sont régulièrement des chômeurs saisonniers (ils seraient couverts par un autre régime de prestations); que ne seraient admis à recevoir des prestations que ceux qui au cours de l'année précédant la première de ces prestations auront versé au moins 20 semaines complètes $^{3}$ de contributions (en plus des 30 semaines complètes pour les deux années précédant la première prestation); et que les gains permis audelà desquels toute prestation sera refusée ou réduite seront diminués.

6 - Tous ces facteurs auront pour effet de réduire les dépenses de la Caisse. D'un autre côté cependant un changement dans les taux de prestations contribuera à les augmenter. Et ce, pour deux raisons: d'abord parce qu'on suggère la création d'une classe nouvelle pour ceux qui gagnent $\$ 80.00$ et plus par semaine; ensuite parce que les taux hebdomadaires de prestations proposés sont plus élevés que les anciens d'un montant variant entre $\$ 2.00$ et $\$ 8.00$ par semaine selon les classes de gains.

(2) Rapport Gill, page 1 .

(3) Présentement certaines semaines sont comptées comme complètes alors qu'elles ne le sont pas. 
7 - Par ailleurs, il faut aussi considérer un changement majeur qui contribuera à l'augmentation des revenus du fonds. Il s'agit de la participation au régime d'assurance-chômage. En principe, tous les employés seront tenus d'y participer sauf ceux pour lesquels le contrôle administratif des contributions serait trop compliqué (exemple: les ouvriers agricoles et les domestiques). C'est ainsi que les instituteurs, les professeurs d'université, les professionnels salariés seront couverts par l'assurance-chômage. Les commissaires sont d'avis que l'exclusion de tels groupes n'est plus fondée. Par ailleurs, ne pourraient participer à lassurance, les personnes de moins de 18 ans. Il est évident que l'exclusion des personnes les plus susceptibles de chômer et l'inclusion des personnes les moins susceptibles de le faire contribueront fortement à régler le problème financier de la Caisse.

8 - En somme, ce problème est relativement facile à régler. Il s'agit de suggérer des règlements qui augmentent les revenus de la Caisse et en diminuent les dépenses. Et le truc est joué. Du même coup les commissaires arrêtent la saignée financière et enlèvent une partie des inquiétudes de ceux qui sont irrégulièrement et temporairement sans travail (moins de 26 semaines).

9 - Mais en réglant ces deux problèmes, les commissaires en créent deux autres. Tout d'abord, ils s'aliénent l'appui de ceux dont le risque d'être irrégulièrement et temporairement en chômage est faible (soit parce que leur risque d'être régulièrement en chômage temporaire (les chômeurs saisonniers) ou celui d'être en chômage prolongé (plus de 26 semaines) est élevé ou que leur risque d'être sans emploi est faible) en les forçant à participer par leurs contributions au paiement des prestations à une catégorie de travailleurs à laquelle ils n'appartiendront peut-être jamais. Ensuite, ils ramènent dans toute sa plénitude le problème de ceux qui sont régulièrement en chômage temporaire et de ceux qui sont en chômage prolongé.

10 - Ils suggèrent alors que le soulagement du problème financier de ces deux dernières catégories de travailleurs relèvent du gouvernement fédéral pour des périodes pouvant aller jusqu'à 39 semaines. De cette façon certains chômeurs pourraient recevoir des prestations pendant un maximum de 65 semaines alors que le maximum actuel est de 52 semaines. La suggestion des commissaires est généreuse. Le revenu des travailleurs sera mieux protégé sous le nouveau régime que sous l'ancien. Mais à ce moment-là, les commissaires - qui viennent tout juste de régler le problème financier de la Caisse - en créent un au gouvernement. Ces nouvelles prestations seront à la charge du gouvernement et payées à même le produit général des impôts. Ce serait ajouter présentement une centaine de millions de dollars aux dépenses du gouvernement fédéral. Il nous semble que cette suggestion des commissaires est heureuse. Elle s'avère en tout cas plus équitable que le régime actuel. Parce que le chômage n'est pas la responsabilité des travailleurs, mais le résultat du fonctionnement d'un régime économique, il est équitable que tous les participants au régime partagent les 
conséquences et non pas seulement ceux qui en subissent le plus durement les contre-coups.

11 - Enfin pour tous ceux qui ne seraient plus couverts par les prestations en provenance de la Caisse ou du gouvernement fédéral, les commissaires suggèrent une aide financière partagée par les gouvernements fédéral et provincial selon la formule actuelle de l'assistance chômage à laquelle on apporterait certaines modifications. Cette dernière catégorie complète le règlement d'une partie du problème financier de ceux à qui le régime économique ne peut fournir du travail pendant des périodes plus ou moins prolongées.

12 - C'est ainsi qu'après avoir totalement réglé le problème financier de la Caisse d'assurance-chômage, les commissaires suggèrent le règlement d'une partie des problèmes financiers de tous les sans-travail. Il reste maintenant à trouver des solutions au problème qui est à la source de ces deux premiers: celui du chômage.

A cet égard, les commissaires offrent au gouvernement une incitation nouvelle au règlement de ce dernier: ses déboursés en prestations seront d'autant plus faibles qu'il aura pris des moyens énergiques pour remédier au chốmage.

Il faut souhaiter que les recommandations des commissaires qui permettent une amélioration importante du régime actuel - soient acceptées au complet et mises en pratique dans le plus bref délai possible.

\section{ECONOMIE ET HUMANISME}

Reowe publike tous les deux mois par le Centre Economie et Humanisme

Sommaire du numéro 141, supplément annuel 1962

Propriété et socialisation

F. Mariney

V. de Couesnongle

J. Lachunal

I. Peranes

P. Vuu

G. Tunar

J. Chaumeny

L.-J. LEBRET
La propriété en question dans l'économie moderne

Morale, régime des biens et socialisation

Morale et biens de production dans une économie socialisée

Grandes entreprises et pouvoir économique

Valeurs et avenir des petites ot mojennes entreprises

Pour une éthique de la répartition

Personne et consommation socialisée

Solidarité internationale et richesses mondiales

Abonnement annuel: \$6.50. Periodica, 5090, Ave Papineau, Montréal 34.

Direction: 99, quai Clémenceau, Caluire, Rhóne, France. 